\title{
The psychenet public health intervention for anorexia nervosa: a pre-post-evaluation study in a female patient sample
}

\author{
Antje Gumz ${ }^{1,{ }^{\dagger}}$, Angelika Weigel ${ }^{2,+}$, Karl Wegscheider $^{3}$, Georg Romer $^{4}$ and Bernd Löwe ${ }^{5}$ \\ ${ }^{1}$ Senior Consultant, Department of Psychosomatic Medicine and Psychotherapy, University Medical Centre \\ Hamburg-Eppendorf \& Schön Clinic Hamburg Eilbek, Hamburg, Germany \\ ${ }^{2}$ Senior Research Fellow, Department of Psychosomatic Medicine and Psychotherapy, University Medical Centre \\ Hamburg-Eppendorf \& Schön Clinic Hamburg Eilbek, Hamburg, Germany \\ ${ }^{3}$ Director, Department of Medical Biometry and Epidemiology, University Medical Centre Hamburg-Eppendorf, \\ Hamburg, Germany \\ ${ }^{4}$ Director, Department of Children and Adolescent Psychiatry, Psychosomatics and Psychotherapy, University Medical \\ Centre Münster, North Rhine-Westphalia, Germany \\ ${ }^{5}$ Director, Department of Psychosomatic Medicine and Psychotherapy, University Medical Centre Hamburg-Eppendorf \\ \& Schön Clinic Hamburg Eilbek, Hamburg, Germany
}

\begin{abstract}
Aim: This non-randomized pre-post-intervention study investigated the effect of a systemic public health intervention on the length of time between anorexia nervosa symptom onset and contact with the health care system as well as the initiation of treatment. Background: Although systemic public health interventions have successfully been implemented in physical and mental health fields, their effect on the early treatment of patients with anorexia nervosa remains unclear. Methods: In total, 59 anorexia nervosa patients (mean age $=21.5$ years, $S D=7.2$ ) were recruited before a systemic public health intervention, and 18 patients (mean age $=22.2$ years, $S D=8.9$ ) were recruited afterwards. Using validated self-report measures and a semi-structured interview, the duration of untreated anorexia nervosa and the duration until first contact with the health care system were investigated. Findings: At the beginning of the individual treatment initiation process, participants in both samples most frequently consulted their general practitioner or paediatrician about their eating disorder-related symptoms. Neither the mean duration of untreated anorexia nervosa, that is, the time between illness onset and the initiation of a recommended treatment, nor the duration until first contact with the health care system significantly decreased after the implementation of the systemic public health intervention. The mean duration of untreated anorexia nervosa was 36.5 months $(S D=68.2)$ before the systemic public health intervention and 40.1 months $(S D=89.4)$ after the implementation of the systemic public health intervention. The mean duration until first contact with the health care system was 25.0 months $(S D=53.0)$ before the intervention and 32.8 months $(S D=86.5)$ after the intervention. Conclusion: Primary care providers are crucial to the treatment initiation process and should be involved in future interventions to improve early detection and treatment commencement amongst patients with anorexia nervosa.
\end{abstract}

Key words: anorexia nervosa; duration of untreated illness; eating disorders; mental health care; public health intervention

Received 3 August 2016; revised 30 June 2017; accepted 16 July 2017; first published online 22 August 2017

Correspondence to: Dr Angelika Weigel, Dipl.-Psych., PhD, Research Fellow, Department of Psychosomatic Medicine and Psychotherapy, University Medical Centre Hamburg, Martinistraße 52, 20246 Hamburg, Germany. Email: a.weigel@uke.de

${ }^{\dagger}$ Antje Gumz and Angelika Weigel contributed equally to this work. 


\section{Introduction}

Anorexia nervosa (AN) is a severe mental disorder that has significant consequences for affected people (Schlegl et al., 2014), their relatives (Coomber and King, 2013) and public health (Stuhldreher et al., 2014). In Europe, $1-4 \%$ of women are affected by AN, with stable incidence rates over the past decade (Keski-Rahkonen and Mustelin, 2016). The physical health impairments associated with AN include cardiac arrhythmias, electrolyte imbalances, renal insufficiency, osteoporosis and severe infections (Agras, 2001). Comorbid mental health disorders are frequent with anxiety, and affective disorders being the most common comorbidities (eg, Herpertz-Dahlmann et al., 2001). The high mortality associated with AN might be attributable to the increased risk for suicide and reasons directly related to the symptoms of AN (Löwe et al., 2001; Smink et al., 2012; Keski-Rahkonen and Mustelin, 2016).

A brief period of untreated AN and a body mass index (BMI) close to the normal range at treatment initiation are strong predictors of a successful treatment outcome and positive prognoses (Löwe et al., 2001; Sly and Bamford, 2011). Consequently, the German and British Guidelines for eating disorders (NICE, 2017; Herpertz et al., 2011) as well as the American Psychiatric Association (2006) stress the importance of early diagnosis and treatment commencement amongst patients with $\mathrm{AN}$ to prevent chronic disease.

The time between illness onset and the initiation of a recommended treatment is the 'duration of untreated illness'. This period has been estimated as 1.78 years on average amongst people with AN (Schoemaker, 1997). Individual and systemic factors influence the duration of untreated AN. Only half of affected individuals report eating disorder symptoms to their general practitioners (Becker et al., 2005). In turn, evidence shows that physicians rarely recognize (Linville et al., 2012) or diagnose AN (Hudson et al., 2013); furthermore, they lack sufficient knowledge about eating disorder treatment (Girz et al., 2014). Even after receiving an eating disorder diagnosis, affected individuals tend to refuse to begin the recommended treatment because of ambivalent attitudes towards treatment and recovery (Leavey et al., 2011), or a lack of knowledge of specialized treatment options (Weigel et al., 2015). Availability and the networking of different health care providers might be deficient (Waller et al., 2009). These limitations may negatively influence individual pathways through the health care system (House et al., 2012).

Public health interventions have been shown to prevent disorders and promote physical and mental health (Kahn et al., 2002; Brousseau et al., 2010; Mears et al., 2014). Successful public health interventions have been designed to reduce suicide rates amongst individuals suffering from depression (Mann et al., 2005; Hübner-Liebermann et al., 2010). However, little research has been conducted with regard to the potential of public health interventions to increase health care use amongst people with eating disorders (Becker et al., 2014).

The present study evaluated the effect of the 'psychenet Healthcare Network Campaign' on the duration between AN symptom onset and the initiation of contact with the health care system as well as the initiation of an evidence-based eating disorder treatment in a female patient population. We hypothesized that after the implementation of this systemic public health intervention, (a) patients would report a briefer duration of untreated AN compared with those whose first treatment occurred before the implementation, and (b) the time between symptom onset and the first contact with the health care system would decrease. In addition, we investigated the type of health care professionals who were patients' first point of contact after the onset of AN symptoms.

\section{Methods}

\section{Participants}

This non-randomized pre-post-intervention study was conducted between 2011 and 2014 across the metropolitan area of Hamburg, Germany. The study compared the duration of untreated AN using two independent crosssectional samples of female patients who were in treatment for the first time. The first patient sample (ie, the 'pre-sample'; recruitment period, January 2012 to February 2013) was assessed before the implementation of the systemic public health intervention. The second sample (ie, the 'post-sample'; recruitment period, November 2013 to May 2014) was assessed after the implementation of the 'psychenet Healthcare Network Primary Health Care Research \& Development 2018; 19: 42-52 


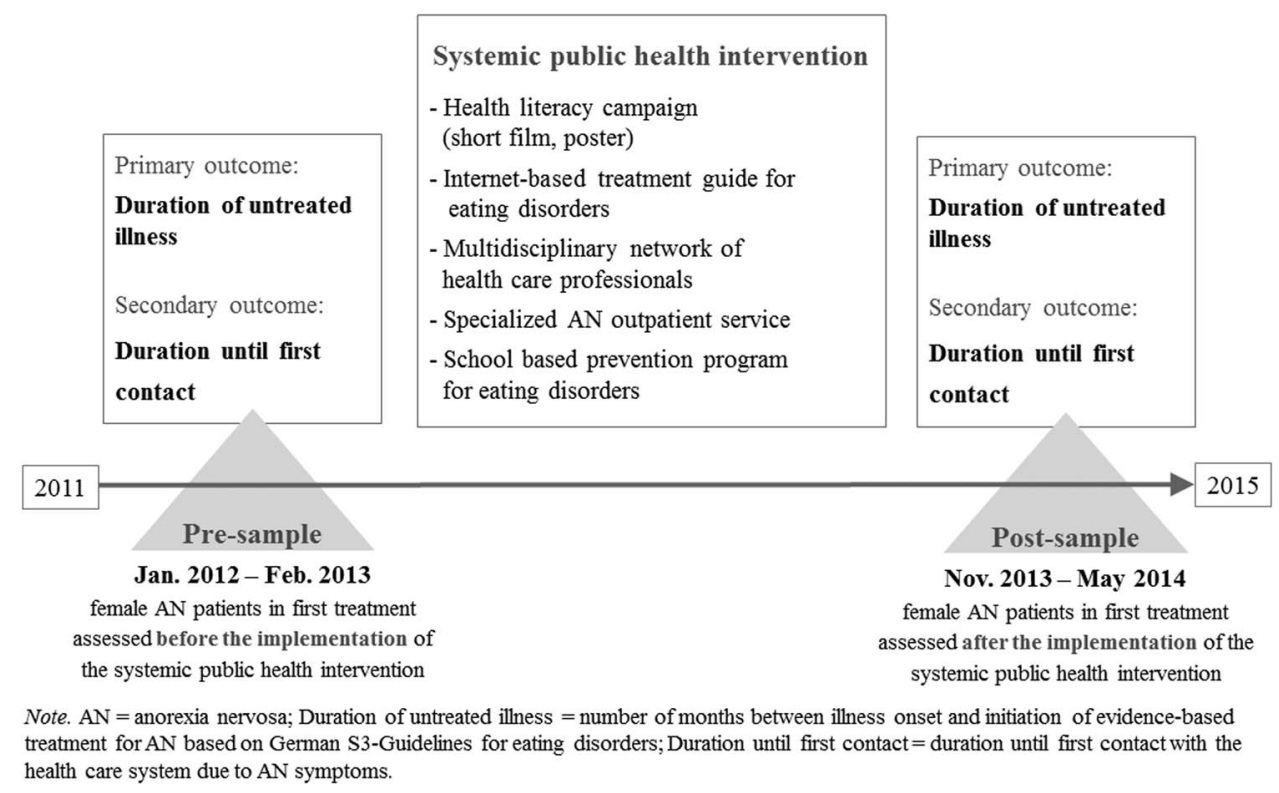

Figure 1 Study design of a community-level systemic public health intervention for patients with anorexia nervosa (AN). Duration of untreated illness $=$ number of months between illness onset and initiation of evidence-based treatment for AN based on German S3-Guidelines for eating disorders; Duration until first contact = duration until first contact with the health care system for eating disorder symptoms.

Campaign'. The trial was registered with an online public registry (ISRCTN44979231; Principal Investigator: B.L.). Figure 1 displays an overview of the study course, elements of the systemic intervention and the study outcomes.

Recruitment over the first three months in the presample indicated that the initial recruitment goal of 100 patients in each sample was not feasible. Therefore, we extended the initial age range from between 12 and 39 years to between 10 and 60 years to sample individuals with (a) particularly early AN onset and (b) an average age of onset but a long duration of untreated AN. In addition, the recruitment period for the pre-sample was extended to 12 months. The project duration prevented an extension of the recruitment period for the post-sample. Additional inclusion criteria for both samples were as follows: female gender, current diagnosis of AN according to the Diagnostic and Statistical Manual of Mental Disorders (DSM-IV) criteria (American Psychiatric Association, 2000), participation in a diagnostic telephone interview, currently undergoing their first eating disorder-specific treatment, and the provision of written informed consent. Eligible participants aged 10-15 years were required to provide written

Primary Health Care Research \& Development 2018; 19: 42-52 informed consent from their parent or legal guardian in addition to their own written informed consent. Participants between the ages of 16 and 18 years are legally able to provide written informed consent and were not required to provide additional consent from a parent or legal guardian. The exclusion criteria were as follows: insufficient German language skills or severe organic or psychological complaints preventing participation (based on the recruiting medical doctors' or psychotherapists' point of view). In addition, participants who did not live in Hamburg were excluded because they were regarded as unable to receive the systemic public health intervention.

\section{Procedure}

The procedure followed our published study protocol (Gumz et al., 2014). Participants were recruited from all available institutions in the Hamburg metropolitan area that offered evidencebased treatment for AN (Herpertz et al., 2011). In total, 11 inpatient wards, one day clinic, 19 outpatient departments and four eating disorderspecific counselling centres were contacted for recruitment. Each of these settings appointed a 
study therapist who was responsible for recruitment. Before recruitment, these study therapists received a preparatory training that included information regarding the overall study, procedure, eligibility criteria, materials and so on. During the recruitment period, the study therapists informed all eligible patients about the study's purpose, obtained written informed consent and distributed the survey package. The survey package was composed of self-report measures assessing prior help-seeking behaviour, current eating disorder pathology, depression and anxiety symptom severity, and additional items regarding age, height, weight and educational level. If the patients met the inclusion criteria, then they were contacted via telephone to participate in a semi-structured diagnostic interview about AN. Participants were regarded as having dropped-out if they refused to participate in the telephone interview or if they could not be contacted after 10 attempts. Participants received a voucher for a free film of their choice at a local cinema in exchange for their participation, and the therapists were given a $€ 7$ online book voucher for each person they successfully referred to the study. The study and consent procedures were reviewed and approved by the ethics committee of the Psychotherapist Chamber of Hamburg.

\section{Systemic public health intervention}

The systemic public health intervention was designed to facilitate the early recognition of $\mathrm{AN}$ and diagnostic and treatment initiation for individuals suffering from AN by reaching affected individuals through their social environments and practitioners. The social environment was addressed with a health literacy campaign that consisted of a brief film presented at regional cinemas and a corresponding poster campaign in the Hamburg region (Figure 1). The film portrayed the personal experiences of a woman suffering from AN. The posters showed a portrait of the woman's eyes, a quotation from the film and a note about the prevalence of $\mathrm{AN}$. This film and the corresponding poster campaign sought to increase awareness of eating disorders and decrease the stigma of mental disorders and faciliate help-seeking.

An internet-based treatment guide for people with eating disorders was developed to reach affected individuals as well as their relatives, peers and health care professionals. The treatment guide provided information about AN, bulimia nervosa and binge eating disorder, treatment guidelines, and the contact information of local inpatient and outpatient institutions specialized in treating eating disorders. Information was adapted for each target group (patients, relatives and practitioners) and available in German, English and Turkish to address individuals with non-German backgrounds.

Practitioners were addressed through the establishment of a multidisciplinary health care network focussing on patients with eating disorders. The network met quarterly, and members of all recruitment centres as well as practitioners from other institutions with a focus on eating disorder participated. Each meeting was composed of a theoretical input from the study team (eg, current treatment guidelines, new therapeutic approaches for AN, medical management of AN), and a network member presented the casework.

Furthermore, a specialised AN outpatient service at the Department of Psychosomatic Medicine and Psychotherapy at the University Medical Centre Hamburg-Eppendorf was implemented. At this centre, affected individuals receive eating disorder diagnoses and treatment recommendations according to the current national guidelines (Herpertz et al., 2011) for eating disorders. Moreover, their primary care providers are provided with suggestions for the accompanying medical management. Lastly, a dissonance-based prevention programme for eating disorders was developed and evaluated within a large randomised controlled trial (for the study protocol, see Weigel et al., 2015). In this programme, all participating schoolchildren and their parents received a flyer including information about treatment options for adolescents suffering from eating disorders. All of the aforementioned elements of the systemic intervention were delivered between March 2013 and December 2014. The distribution of project flyers, talks at different public events, interviews in local newspapers and advertisements in journals for psychotherapists and medical practitioners were used as dissemination strategies. A more detailed description of the 'psychenet Healthcare Network Campaign' can be found elsewhere (Gumz et al., 2014).

\section{Measures}

The 'duration of untreated illness' was the primary study outcome and was operationalized as Primary Health Care Research \& Development 2018; 19: 42-52 
the number of months between AN symptom onset and the commencement of an evidencebased treatment, based on the German S3-Guidelines for eating disorders (Herpertz et al., 2011). During the telephone interview, AN was diagnosed using the eating disorder section of the Structured Clinical Interview for DSM-IV (Wittchen et al., 1997). Therein, the onset of each diagnostic criterion for AN was specified. The date when all AN criteria were first met was operationalized as the illness onset. To quantify the individual duration of untreated $\mathrm{AN}$ in months, the date of the first eating disorderspecific treatment was recorded.

'Duration until first contact with the healthcare system' was the secondary study outcome and was assessed with a self-report measure that was part of the survey package. This variable was included to improve the understanding of the interval preceding the initiation of an evidence-based treatment for AN (House et al., 2012). It was operationalized as the number of months between illness onset and first contact with a health care professional for eating disorder-related symptoms. This variable might indicate patients' help-seeking behaviours before beginning a specialized treatment. A questionnaire developed for the current study comprised different potential health care professionals that were contacted for eating disorder-related symptoms (eg, general practitioner, gynaecologist, dentist, psychotherapist and so on). In this questionnaire, participants indicated (a) whether they had contact with one or more of these health care practitioners, and (b) the year and month of the first contact with each health care professional.

To compare the current eating pathology of both samples, the German version of the Eating Disorder Examination-Questionnaire (EDE-Q; Hilbert et al., 2007) or its version for participants younger than 16 years of age (Ch-EDE-Q; Hilbert et al., 2008) was applied. Both self-report measures have satisfactory psychometric properties (Hilbert et al., 2012). Current depression and anxiety symptom severity, the most common AN comorbidities, were collected to further characterise the study sample. These variables were assessed using the German versions of the nine-item depression module of the Patient Health Questionnaire (Spitzer et al., 1999; Löwe et al., 2004a; 2004b) and the Generalized Anxiety Disorder Scale (Löwe et al., 2008; Kroenke et al., 2010; Wild et al., 2014), respectively. Both instruments exhibited acceptable reliability and validity (Kroenke et al., 2001; Löwe et al., 2004b, Spitzer et al., 2006; Löwe et al., 2008). Age, weight, height and educational level were also assessed as part of the questionnaire.

\section{Statistical methods}

Based on previous studies (Schoemaker, 1997) with an average duration of untreated $\mathrm{AN}$ of 19 months and an estimated effect size of Cohen's $\mathrm{d}=0.4$, we considered a decrease of untreated AN of 4.8 months or $25 \%$ as clinically relevant. Thus, when conducting linear mixed-model analyses, a two-tailed type-I error of $5 \%$, and a power of $80 \%$, two samples with 100 participants each were required.

The data were analysed using IBM SPSS Statistics for Windows, version 21 (IBM Corp., Armonk, NY, USA). We conducted two-tailed independentsamples $t$-tests and $\chi^{2}$-tests for nominally scaled and non-parametric variables to compare both crosssectional samples with regard to participant characteristics and levels of observed psychopathology. Frequencies are reported for answers to the variable 'member of the health care system first contacted for eating disorder symptoms'.

Before conducting the linear mixed-model analyses, numerical data were inspected for normality using histograms. 'Duration of untreated illness' and 'duration until first contact with the health care system' were skewed and were consequently logarithmically transformed. The primary hypothesis (ie, whether differences exist between the pre- and post-intervention measures of the mean 'duration of untreated illness') was tested by applying a linear mixed-model analysis using maximum likelihood estimation. The period of assessment was specified as the independent variable, and the duration of untreated illness was the primary endpoint. Recruitment setting was included as a random control variable, and age was included as a fixed control variable.

As a secondary analysis, we calculated an analogous linear mixed-model with the mean 'duration until first contact with the health care system' as the dependent variable. In each case, $95 \%$ confidence intervals (CIs) were used to express the uncertainty in the data. As no variable showed missing values over $5 \%$, the analyses were performed using complete cases and were not imputed. Two-tailed $P$-values $<0.05$ were considered as significant. 


\section{Results}

\section{Patient sample characteristics}

The participation rates were $60.8 \%$ for the pre-intervention and $50.0 \%$ for the postintervention groups. Figure 2 illustrates the flow of patients before and after the implementation of the systemic public health intervention.

Both cross-sectional samples were well balanced with respect to participant characteristics and levels of observed psychopathology (Table 1). The majority of patients in both samples met the diagnostic criteria for the restrictive subtype of AN according to the DSM-IV. Based on the DSM-5 criteria for full threshold AN (American Psychiatric Association, 2013), 74.6\% $(n=44)$ of the patients in the pre-intervention group and $94.4 \%$ of the patients in the post-intervention group $(n=17)$ would have received this diagnosis.

\section{Evaluation of the systemic intervention}

The mean duration of untreated AN was 36.5 months ( $\mathrm{SD}=68.2$, range $1-438$ months) before the intervention and 40.1 months $(\mathrm{SD}=89.4$, range 3-393 months) after the implementation of the systemic public health intervention. The mean duration until first contact with the health care system was 25.0 months $(\mathrm{SD}=53.0$, range: -6 to 313 months) before the intervention and 32.8 months ( $\mathrm{SD}=86.5$, range 1-375 months) after intervention. Thus, neither the duration of untreated AN (adjusted mean difference $=0.07$ months, $95 \%$ CIs -0.18 to $0.32, P=0.58$ ) nor the duration until first contact with a health care professional (adjusted mean difference $=0.08$ months, $95 \%$ CI -0.37 to 0.20 , $P=0.57)$ significantly decreased from pre- to postassessment amongst women who received treatment for AN for the first time (Figure 3).

At the beginning of the individual treatment initiation process, participants in both samples most frequently consulted their general practitioner or paediatrician for their eating disorder-related symptoms $\left(\chi^{2}=4.86, \mathrm{df}=2, P=0.71\right.$; Figure 4$)$.

Within the post-intervention group (ie, participants assessed after the implementation of the systemic intervention), $16.7 \% \quad(n=3)$ of the patients were aware of the brief film that had been shown in cinemas. Another $22.2 \%(n=4)$ had visited the main psychenet homepage and the internet-based treatment guide. Finally, 22.2\% $(n=4)$ had used the specialised AN outpatient

assessed before implementation of public health intervention

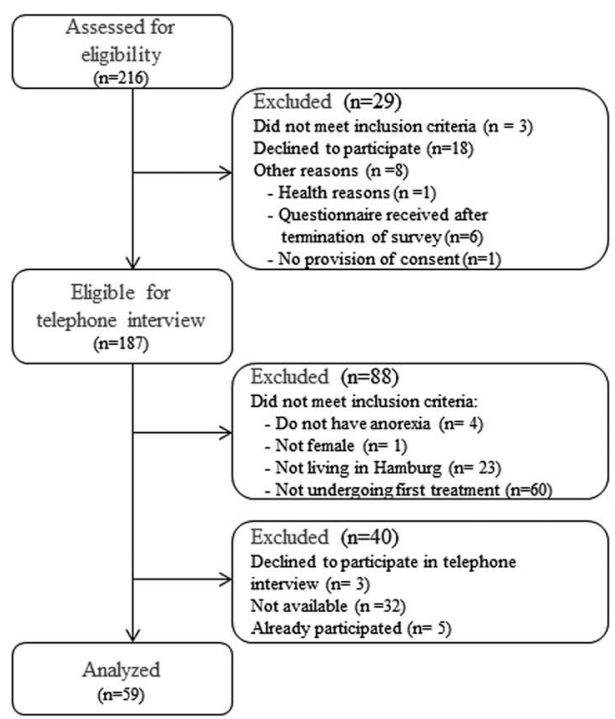

Post-sample



Figure 2 Flowchart of female anorexia patients in first treatment assessed before and after the implementation of a community-level systemic public health intervention. Participants who were not living in Hamburg were excluded because they were regarded as not having received the systemic public health intervention. 
Table 1 Characteristics of female patients with anorexia nervosa (AN) undergoing their first specialized anorexia treatment pre- and post-implementation of a systemic public health intervention

\begin{tabular}{|c|c|c|c|c|}
\hline & Pre-sample $(n=59)$ & Post-sample $(n=18)$ & Statistic & $P$-value \\
\hline Current age (mean and SD) & $21.5(7.2)$ & $22.2(8.9)$ & $t=-0.33$ & 0.74 \\
\hline Age of onset (mean and SD) & $18.0(5.6)$ & $18.8(5.6)$ & $t=-0.58$ & 0.56 \\
\hline AN subtype ( $\%$ and $n$ restrictive) & $56.9(33)$ & $72.2(13)$ & $\chi^{2}=5.94$ & 0.12 \\
\hline Setting of first treatment ( $\%$ and $n$ inpatient) & $55.9(33)$ & $83.3(15)$ & $\chi^{2}=4.86$ & 0.09 \\
\hline Educational level (\% and $n$ higher) & $68.6(24)$ & $75.0(9)$ & $\chi^{2}=4.88$ & 0.30 \\
\hline Current BMI (mean and SD) & $17.2(1.7)$ & $16.9(1.3)$ & $t=0.66$ & 0.51 \\
\hline EDE-Q (global mean score and SD) & $3.6(1.3)$ & $3.6(1.6)$ & $t=-0.95$ & 0.35 \\
\hline PHQ-9 (global sum score and SD) & $13.0(5.7)$ & $14.7(6.6)$ & $t=1.10$ & 0.28 \\
\hline GAD-7 (global sum score and SD) & $9.8(4.9)$ & $10.9(5.8)$ & $t=-0.82$ & 0.41 \\
\hline
\end{tabular}

Pre-sample $=$ female AN patients in first treatment assessed before the implementation of the systemic public health intervention; Post-sample = female AN patients in first treatment assessed after the implementation of the systemic public health intervention; $\mathrm{BMI}=$ body mass index; $\mathrm{EDE}-\mathrm{Q}=$ Eating Disorder Examination-Questionnaire; $\mathrm{PHQ}-9=$ depression module of the Patient Health Questionnaire; GAD-7 = anxiety module of the Patient Health Questionnaire. Educational level = 'higher' level of education - at least a general qualification to begin university.

Two-tailed $t$-tests for independent samples were used for metric variables as well as $\chi^{2}$-tests for nominal scaled and nonparametric variables. Two-tailed $P$-values $<0.05$ were considered significant.

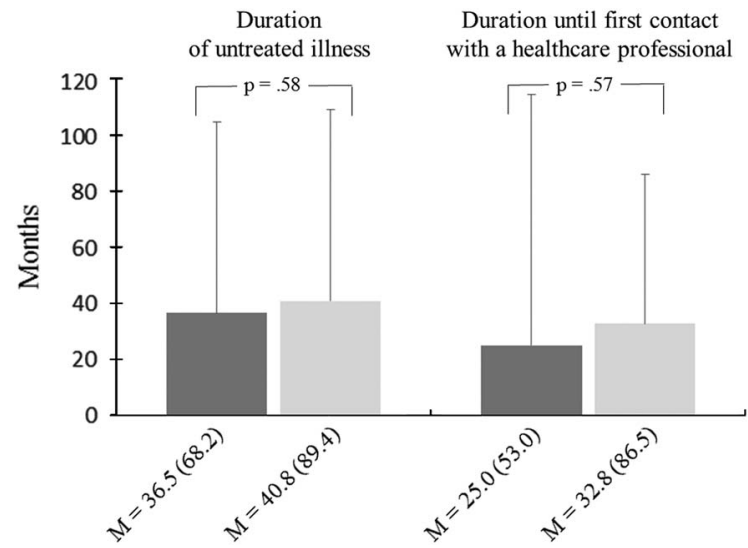

Figure 3 Duration of untreated illness and duration until first contact with a health care professional in female patients with anorexia nervosa before and after the implementation of a systemic public health intervention. Dark grey bars reflect the results of the pre-intervention group, and light grey bars reflect the results of the postintervention group. Standard deviation in brackets. $M=$ mean.

clinic at the Department of Psychosomatic Medicine and Psychotherapy, University Medical Centre Hamburg-Eppendorf.

\section{Discussion}

The primary aim of the present study was to evaluate the effect of a systemic community-level Primary Health Care Research \& Development 2018; 19: 42-52 public health intervention on the duration from AN onset to the initiation of contact with the health care system as well as the initiation of evidence-based eating disorder treatment in a sample of women with AN.

Before the implementation of the intervention, more than three years passed on average between the onset of AN and treatment initiation. Against our expectations, the time span remained statistically unchanged after the implementation of the systemic public health intervention. Likewise, the 'psychenet Healthcare Network Campaign' did not affect the time span between symptom onset and first contact with a health care professional.

The average duration of untreated AN observed in this study was considerably longer than the average duration of untreated AN according to a previous review (19 months; Schoemaker, 1997). The inclusion of adolescent and adults with AN in the present study and the consideration of both in- and outpatient sectors might explain these differences. From a clinician's point of view, these results are alarming and underline the need to improve early diagnosis and treatment amongst patients with AN to prevent affected individuals from chronic disease and the severe complications of this disorder (Herzog et al., 1997; Löwe et al., 2001).

The possible reasons for why the 'psychenet Healthcare Network Campaign' did not improve early treatment for $\mathrm{AN}$ include the following. 




Figure 4 Health care professional first visited by female patients with anorexia nervosa because of eating disorder symptoms before treatment initiation. $n_{1}=58$ patients assessed before the implementation of a community-level systemic public health intervention; $n_{2}=18$ patients assessed after the implementation. Bar charts reflect the percentages of settings indicated $n>5$. Settings indicated $<5$ were not included (eg, neurologist, dermatologist, endocrinologist, dentist, non-medical practitioner and nutritionist). GP=general practitioner.

First, the sample size might have been too small to capture the effects. Second, it is possible that the intervention as a whole was ineffective. Third, the intervention might not have reached the relevant target groups. Fourth, the implementation period might have been too brief to allow for changes in the duration of untreated AN to become apparent. Finally, systemic community-level public health interventions in general might not be an appropriate tool to reach patients with $\mathrm{AN}$ during the early phase of their disorder.

We were unable to achieve our recruitment goal of 100 patients in each sample. This limitation is the greatest of our study because it decreases the interpretability of our results. In the pre-intervention group, we attempted to reach our recruitment goal by extending the age range and the recruitment period. As a consequence, it was necessary to compromise between a sufficiently long blanking period between the pre- and post-intervention groups in which the public health intervention was implemented and a sufficiently long recruitment period for the post-intervention group before the end of the study. This compromise resulted in different sample sizes of the two groups. The low prevalence of AN impedes the assessment of large samples in this population (Jacobi et al., 2014). Nevertheless, we assessed the eligibility of 336 patients with AN over the course of the study. Of these patients, many were not experiencing their first treatment and therefore did not meet the inclusion criteria. If we had included patients with AN whose first treatment occurred in the past, then the sample size and associated statistical power would have been higher. However, such a change might have decreased the internal validity of our study because of a potential recall bias.

The second major drawback of the present study is that the exposure to the intervention could not be evaluated. This issue is common amongst public health interventions (Craig et al., 2008). Therefore, we cannot determine whether the intervention was not effective because it did not diffuse sufficiently to reach all relevant community members or whether the intervention period was too brief to affect the relevant participants.

In addition, randomly assigning patients to different groups was not considered as feasible. As the intervention was conducted across the Hamburg metropolitan area, randomly allocating eligible participants to one group receiving the intervention and another group (ie, the control group) not receiving the intervention was not possible. However, we attempted to overcome this limitation by assessing one sample of patients before the implementation of the intervention and

Primary Health Care Research \& Development 2018; 19: 42-52 
the other after implementation. Future studies evaluating the effect of public health interventions on early treatment among patients with AN should apply a randomized controlled design with a sufficiently large sample to increase internal validity (Becker et al., 2014).

In addition, the variables of interest (eg, information about first treatment) were primarily assessed through retrospective self-reports and are vulnerable to inaccuracies. To counteract this possible recall bias, self-reported data were reviewed during the telephone interview. Importantly, a prospective assessment of these variables was not feasible given the low prevalence of AN. In addition, BMI was assessed through self-reports, which might have resulted in overestimation. However, the self-reported weight assessed during the telephone interview referred to the weekly mandatory weight measurements during the inpatient or outpatient treatments.

Patients with extremely low body weights were excluded from our sample when cognitive deficits prevented them from completing the self-report measures or from participating in the telephone interview. These patients might display a particularly long duration of untreated AN.

Finally, our sample only included women. We decided to focus on this gender to achieve homogeneity within the sample. However, conclusions drawn from our results are not transferable to men who suffer from AN.

\section{Implications of research}

Our study was the first to investigate the influence of a community-level systemic public health intervention on treatment initiation amongst people with AN. Given the high prognostic value of early treatment and the mean duration of untreated $\mathrm{AN}$ of more than three years, our results underline the need for an improved understanding of the process of treatment initiation amongst people with AN to develop effective interventions and prevent affected individuals from severe, chronic disease.

In line with prior evidence, our results emphasized that primary caregivers are an important interface on the path towards evidence-based AN treatment (Kessler, 2009). Future public health interventions to improve early diagnosis and treatment for people with AN might benefit from a collaboration with primary caregivers (Martin-

Primary Health Care Research \& Development 2018; 19: 42-52
Misener et al., 2012). However, previous studies showed that primary caregivers might suffer from a lack of knowledge regarding the recognition, diagnosis and management of eating disorders (Linville et al., 2012; Hudson et al., 2013; Girz et al., 2014). Shared care attempts based on a multiprofessional collaboration between primary care providers and mental health professionals might facilitate the recognition, diagnosis and management of AN in primary care. Although shared care models have revealed promising results in the areas of maternal mental health (Li et al., 2016) as well as depression and anxiety (Archer et al., 2012) evidence from the eating disorder field is still needed. A systemic qualitative review of enablers and the barriers to implementing collaborative care for patients with anxiety and depression (Overbeck et al., 2016) indicated that future shared care attempts for patients with AN should put particular emphases on (a) effective educational programs, (b) appropriate reimbursements for extra work related to primary care providers, (c) the establishment of effective systems to ensure communication and monitoring between stakeholders and (d) the promotion of regular face-to-face interactions between care managers and primary care providers.

\section{Implications for practice}

- The mean duration of untreated AN exceeds three years.

- Help-seeking in patients with AN most frequently begins with the general practitioner.

- Evidence-based treatment does not typically begin until one year after the initial helpseeking event.

\section{Acknowledgement}

The authors would like to thank Anne Daubmann for her comments on this manuscript.

\section{Financial Support}

The study was part of a health service research project (psychenet) that was funded by the German Federal Ministry of Education and Research (ISRCTN44979231; subproject Anorexia and Bulimia Nervosa; Principal Investigator: B.L.). 


\section{Conflicts of Interest}

None.

\section{Ethical Standards}

The study protocol and consent procedure were reviewed and approved by the ethics committee of the Psychotherapist Chamber of Hamburg. The authors also assert that all procedures contributing to this work comply with the Helsinki Declaration of 1975 as revised in 2008.

\section{References}

Agras, W.S. 2001: The consequences and costs of the eating disorders. Psychiatric Clinics of North America 24, 371-79.

American Psychiatric Association. 2000: Diagnostic and statistical manual of mental disorders - DSM-IV-TR. Washington, DC: American Psychiatric Association.

American Psychiatric Association. 2006: Practice guidelines for the treatment of patients with eating disorders, third edition. New York: American Psychiatric Association.

American Psychiatric Association. 2013: Diagnostic and statistical manual of mental disorders, fifth edition. Washington, DC: American Psychiatric Association.

Archer, J., Bower, P., Gilbody, S., Lovell, K., Richards, D., Gask, L., Dickens, C. and Coventry, P. 2012: Collaborative care for depression and anxiety problems. Cochrane Database Systematic Reviews 10, CD006525.

Becker, A.E., Thomas, J.J., Franko, D.L. and Herzog, D.B. 2005: Disclosure patterns of eating and weight concerns to clinicians, educational professionals, family, and peers. International Journal of Eating Disorders 38, 18-23.

Becker, C.B., Plasencia, M., Kilpela, L.S., Briggs, M. and Stewart, T. 2014: Changing the course of comorbid eating disorders and depression: what is the role of public health interventions in targeting shared risk factors? Journal of Eating Disorders 2, 15.

Brousseau, N., Sauvageau, C., Ouakki, M., Audet, D., Kiely, M., Couture, C., Pare, A. and Deceuninck, G. 2010: Feasibility and impact of providing feedback to vaccinating medical clinics: evaluating a public health intervention. BMC Public Health 10, 750.

Coomber, K. and King, R. M. 2013: Perceptions of carer burden: differences between individuals with an eating disorder and their carer. Eating Disorders 21, 26-36.

Craig, P., Dieppe, P., Macintyre, S., Michie, S., Nazareth, I. and Petticrew, M., Medical Research Council Guidance. 2008: Developing and evaluating complex interventions: the new Medical Research Council guidance. BMJ 337, a1655.

Girz, L., Robinson, A.L. and Tessier, C. 2014: Is the next generation of physicians adequately prepared to diagnose and treat eating disorders in children and adolescents? Eating Disorders 22, 375-85.
Gumz, A., Uhlenbusch, N., Weigel, A., Wegscheider, K., Romer, G. and Löwe, B. 2014: Decreasing the duration of untreated illness for individuals with anorexia nervosa: study protocol of the evaluation of a systemic public health intervention at community level. BMC Psychiatry 14, 300.

Herpertz, S., Herpetz-Dahlmann, B., Fichter, M., Tuschen-Caffier, B. and Zeeck, A. (editors) 2011. S3Leitlinie: Diagnostik und Behandlung der Essstörungen (S3 guideline: the diagnosis and treatment of eating disorders). Heidelberg: Springer.

Herpertz-Dahlmann, B., Müller, B., Herpertz, S., Heussen, N., Hebebrand, J. and Remschmidt, H. 2001: Prospective 10-year follow-up in adolescent anorexia nervosa - course, outcome, psychiatric comorbidity, and psychosocial adaptation. Journal of Child Psychology and Psychiatry 42, 603-12.

Herzog, W., Deter, H.C., Fiehn, W. and Petzold, E. 1997: Medical findings and predictors of long-term physical outcome in anorexia nervosa: a prospective, 12-year follow-up study. Psychological Medicine 27, 269-79.

Hilbert, A., De Zwaan, M. and Brähler, E. 2012: How frequent are eating disturbances in the population? Norms of the eating disorder examination-questionnaire. Plos One 7, e29125.

Hilbert, A., Hartmann, A.S. and Czaja, J. 2008: Child Eating Disorder Examination-Questionnaire: Evaluation der deutschsprachigen Version des Essstörungsfragebogens für Kinder (The Eating Disorder Examination-Questionnaire for children: psychometric properties of the German version). Klinische Diagnostik und Evaluation 1, 447-64.

Hilbert, A., Tuschen-Caffier, B., Karwautz, A., Niederhofer, H. and Munsch, S. 2007: Eating disorder examination-questionnaire: psychometric properties of the German version. Diagnostica 53, 144-54.

House, J., Schmidt, U., Craig, M., Landau, S., Simic, M., Nicholls, D., Hugo, P., Berelowitz, M. and Eisler, I. 2012: Comparison of specialist and nonspecialist care pathways for adolescents with anorexia nervosa and related eating disorders. International Journal of Eating Disorders 45, 949-56.

Hübner-Liebermann, B., Neuner, T., Hegerl, U., Hajak, G. and Spiessl, H. 2010: Reducing suicides through an alliance against depression? General Hospital Psychiatry 32, 514-18.

Hudson, L.D., Cumby, C., Klaber, R.E., Nicholls, D.E., Winyard, P.J. and Viner, R.M. 2013: Low levels of knowledge on the assessment of underweight in children and adolescents among middle-grade doctors in England and Wales. Archives of Disease in Childhood 98, 309-11.

Jacobi, F., Hofler, M., Strehle, J., Mack, S., Geschler, A., Scholl, L., Busch, M.A., Maske, U., Hapke, U., Gaebel, W., Maier, W., Wagner, M., Zielasek, J. and Wittchen, H.U. 2014: Mental disorders in the general population: study on the health of adults in Germany and the additional module mental health (DEGS1-MH). Nervenarzt 85, 77-87.

Kahn, E.B., Ramsey, L.T., Brownson, R.C., Heath, G.W., Howze, E.H., Powell, K.E., Stone, E.J., Rajab, M.W. and Corso, P. 2002: The effectiveness of interventions to increase physical activity. A systematic review. American Journal of Preventive Medicine 22, 73-107.

Primary Health Care Research \& Development 2018; 19: 42-52 
Keski-Rahkonen, A. and Mustelin, L. 2016: Epidemiology of eating disorders in Europe: prevalence, incidence, comorbidity, course, consequences, and risk factors. Current Opinion in Psychiatry 29, 340-45.

Kessler, R. 2009: Across the great divide: introduction to the special issue on psychology in medicine. Journal of Clinical Psychology 65, 231-34.

Kroenke, K., Spitzer, R.L. and Williams, J.B. 2001: The PHQ-9 - validity of a brief depression severity measure. Journal of General Internal Medicine 16, 606-13.

Kroenke, K., Spitzer, R.L., Williams, J.B. and Löwe, B. 2010: The Patient Health Questionnaire Somatic, Anxiety, and Depressive Symptom Scales: a systematic review. General Hospital Psychiatry 32, 345-59.

Leavey, G., Vallianatou, C., Johnson-Sabine, E., Rae, S. and Gunputh, V.2011: Psychosocial barriers to engagement with an eating disorder service: a qualitative analysis of failure to attend. Eating Disorders 19, 425-40.

Li, H., Bowen, A., Szafron, M., Moraros, J. and Muhajarine, N. 2016: Maternal mental health: a shared care approach. Primary Health Care Research \& Development 17, 175-83.

Linville, D., Brown, T. and O'Neil, M. 2012: Medical providers' self-perceived knowledge and skills for working with eating disorders: a national survey. Eating Disorders 20,1-13.

Löwe, B., Decker, O., Müller, S., Brähler, E., Schellberg, D., Herzog, W. and Herzberg, P.Y. 2008: Validation and standardization of the Generalized Anxiety Disorder Screener (GAD-7) in the general population. Medical Care 46, 266-74.

Löwe, B., Gräfe, K., Zipfel, S., Witte, S., Lörch, B. and Herzog, W. 2004a: Diagnosing ICD-10 depressive episodes: superior criterion validity of the Patient Health Questionnaire. Psychotherapy and Psychosomatics 73, 386-90.

Löwe, B., Spitzer, R.L., Gräfe, K., Kroenke, K., Quenter, A., Zipfel, S., Buchholz, C., Witte, S. and Herzog, W. 2004b: Comparative validity of three screening questionnaires for DSM-IV depressive disorders and physicians' diagnoses. Journal of Affective Disorders 78, 131-40.

Löwe, B., Zipfel, S., Buchholz, C., Dupont, Y., Reas, D.L. and Herzog, W. 2001: Long-term outcome of anorexia nervosa in a prospective 21-year follow-up study. Psychological Medicine 31, 881-90.

Mann, J.J., Apter, A., Bertolote, J., Beautrais, A., Currier, D., Haas, A., Hegerl, U., Lonnqvist, J., Malone, K., Marusic, A., Mehlum, L., Patton, G., Phillips, M., Rutz, W., Rihmer, Z., Schmidtke, A., Shaffer, D., Silverman, M., Takashi, Y., Varnik, A., Wasserman, D., Yip, P. and Hendin, H. 2005: Suicide prevention strategies: a systematic review. JAMA 294, 2064-74.

Martin-Misener, R., Valaitis, R., Wong, S.T., MacDonald, M., Meagher-Stewart, D., Kaczorowski, J., O-Mara, L., Savage, R. and Austin, P., The Strengthening Primary Health Care through Public Health and Primary Care Collaborations Team. 2012: A scoping literature review of collaboration between primary care and public health. Primary Health Care Research \& Development 13, 327-46.

Primary Health Care Research \& Development 2018; 19: 42-52
Mears, J., Abubakar, I., Crisp, D., Maguire, H., Innes, J.A., Lilley, M., Lord, J., Cohen, T., Borgdorff, M.W., Vynnycky, E., McHugh, T.D. and Sonnenberg, P. 2014: Prospective evaluation of a complex public health intervention: lessons from an initial and follow-up cross-sectional survey of the tuberculosis strain typing service in England. BMC Public Health 14, 1023.

National Institute for Health and Care Excellence (NICE) 2017. Eating disorders: recognition and treatment. NICE guideline [NG69]: London: NICE Guideline.

Overbeck, G., Davidsen, A.S. and Kousgaard, M.B. 2016: Enablers and barriers to implementing collaborative care for anxiety and depression: a systematic qualitative review. Implementation Science 11, 165.

Schlegl, S., Quadflieg, N., Löwe, B., Cuntz, U. and Voderholzer, U. 2014: Specialized inpatient treatment of adult anorexia nervosa: effectiveness and clinical significance of changes. BMC Psychiatry 14, 258.

Schoemaker, C. 1997: Does early intervention improve the prognosis in anorexia nervosa? A systematic review of the treatment-outcome literature. International Journal of Eating Disorders 21, 1-15.

Sly, R. and Bamford, B. 2011: Why are we waiting? The relationship between low admission weight and end of treatment weight outcomes. European Eating Disorder Review 19, 407-10.

Smink, F.R., van Hoeken, D. and Hoek, H.W. 2012: Epidemiology of eating disorders: incidence, prevalence and mortality rates. Current Psychiatry Reports 14, 406-14.

Spitzer, R.L., Kroenke, K. and Williams, J.B. 1999: Validation and utility of a self-report version of PRIME-MD - the PHQ primary care study. JAMA 282, 1737-744.

Spitzer, R.L., Kroenke, K., Williams, J.B. and Löwe, B. 2006: A brief measure for assessing generalized anxiety disorder: the GAD-7. Archives of Internal Medicine 166, 1092-97.

Stuhldreher, N., Wild, B., König, H.H., Konnopka, A., Zipfel, S. and Herzog, W. 2014: Determinants of direct and indirect costs in anorexia nervosa. International Journal of Eating Disorders 48, 139-46.

Waller, G., Schmidt, U., Treasure, J., Murray, J., Aleyna, J., Emanuelli, F., Crockett, J. and Yeomans, M. 2009: Problems across care pathways in specialist adult eating disorder services. Psychiatric Bulletin 33, 26-29.

Weigel, A., Gumz, A., Uhlenbusch, N., Wegscheider, K., Romer, G. and Löwe, B. 2015: Preventing eating disorders with an interactive gender-adapted intervention program in schools: study protocol of a randomized controlled trial. BMC Psychiatry 15, 21.

Wild, B., Eckl, A., Herzog, W., Niehoff, D., Lechner, S., Maatouk, I., Schellberg, D., Brenner, H., Müller, H. and Löwe, B. 2014: Assessing generalized anxiety disorder in elderly people using the GAD-7 and GAD-2 scales: results of a validation study. The American Journal of Geriatric Psychiatry 22, 1029-38.

Wittchen, H.U., Zaudig, M. and Fydrich, T. 1997. Strukturiertes Klinisches Interview für DSM-IV. Göttingen: Hogrefe. 